\title{
EHR phenotyping via jointly embedding medical concepts and words into a unified vector space
}

\author{
Tian Bai ${ }^{1}$, Ashis Kumar Chanda ${ }^{1}$, Brian L. Egleston ${ }^{2}$ and Slobodan Vucetic ${ }^{1 *}$ \\ From IEEE International Conference on Bioinformatics and Biomedicine 2017 \\ Kansas City, MO, USA. 13-16 November 2017
}

\begin{abstract}
Background: There has been an increasing interest in learning low-dimensional vector representations of medical concepts from Electronic Health Records (EHRs). Vector representations of medical concepts facilitate exploratory analysis and predictive modeling of EHR data to gain insights about the patterns of care and health outcomes. EHRs contain structured data such as diagnostic codes and laboratory tests, as well as unstructured free text data in form of clinical notes, which provide more detail about condition and treatment of patients.

Methods: In this work, we propose a method that jointly learns vector representations of medical concepts and words. This is achieved by a novel learning scheme based on the word2vec model. Our model learns those relationships by integrating clinical notes and sets of accompanying medical codes and by defining joint contexts for each observed word and medical code.

Results: In our experiments, we learned joint representations using MIMIC-III data. Using the learned representations of words and medical codes, we evaluated phenotypes for 6 diseases discovered by our and baseline method. The experimental results show that for each of the 6 diseases our method finds highly relevant words. We also show that our representations can be very useful when predicting the reason for the next visit.

Conclusions: The jointly learned representations of medical concepts and words capture not only similarity between codes or words themselves, but also similarity between codes and words. They can be used to extract phenotypes of different diseases. The representations learned by the joint model are also useful for construction of patient features.
\end{abstract}

Keywords: Electronic health records, Distributed representation, Natural language processing, Healthcare

\section{Background}

Electronic health record (EHR) systems are used by medical providers to streamline the workflow and enable sharing of patient data among providers. Beyond that primary purpose, EHR data have been used in healthcare research for exploratory and predictive analytics in problems such as risk prediction [1-3] and retrospective epidemiologic studies [4-6]. Important challenges in those studies include cohort identification [7, 8], which refers

\section{*Correspondence: vucetic@temple.edu}

'Department of Computer \& Information Sciences, Temple University, Philadelphia, PA, USA

Full list of author information is available at the end of the article to finding a set of patients receiving a specific treatment or having a specific diagnosis, and patient phenotyping $[9,10]$, which refers to identifying conditions and treatments for given diseases from patients' longitudinal records.

EHR data are heterogeneous collections of both structured and unstructured information. In order to store data in a structured way, several ontologies have been developed to describe diagnoses and treatments, among which the most popular coding classification systems is the International Classification of Diseases (e.g, ICD-9, ICD-10). ICD codes provide alpha-numeric encoding of patient conditions and treatments. On the other hand, the

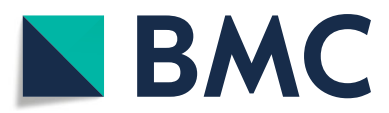

(c) The Author(s). 2018 Open Access This article is distributed under the terms of the Creative Commons Attribution 4.0 International License (http://creativecommons.org/licenses/by/4.0/), which permits unrestricted use, distribution, and reproduction in any medium, provided you give appropriate credit to the original author(s) and the source, provide a link to the Creative Commons license, and indicate if changes were made. The Creative Commons Public Domain Dedication waiver (http://creativecommons.org/publicdomain/zero/1.0/) applies to the data made available in this article, unless otherwise stated. 
unstructured clinical notes contain various more nuanced information (e.g, the history of patient's illness and medication), which creates challenges for designing effective algorithms to transform data into meaningful representations that can be efficiently interpreted and used in health care applications. Various studies manage to discover knowledge from free-text clinical notes. Wang et al. proposed a token matching algorithm to map medical expressions in clinical notes into a structured medical terminology [11]. Pivovarov et al. developed a probabilistic graphical model to infer phenotypes described by medical codes, words and other clinical observations [12]. Joshi et al. proposed a non-negative matrix factorization method to generate latent factors defined by clinical words [13].

The success of extracting knowledge from clinical notes often requires application of Natural Language Processing (NLP) techniques. Learning distributed representations of words using models based on neural networks has been shown to be very useful in many NLP tasks. These models represent words as vectors and place vectors of words that occur in similar contexts in a neighborhood of each other. Among the existing models, Mikolov's word2vec model [14] is among the most popular due to its simplicity and effectiveness in learning word representations from a large amount of data. Several studies applied word2vec on clinical notes data to produce effective clinical word representations for various applications [15-21].

While word2vec was initially designed for handling text, recent studies demonstrate that word2vec could learn representations of other types of data, including medical codes from EHR data [21-25]. Choi et al. used word2vec to learn the vector representations of medical codes using longitudinal medical records and show that the related codes indeed have similar vector representations [22]. Choi et al. designed a multi-layer perceptron to learn representations of medical codes for predicting future clinical events and clinical risk groups [23]. Gligorijevic et al. used word2vec to phenotype sepsis patients [25] and Choi et al. fed code representation learned by word2vec into a recurrent neural network to predict heart failure [24]. The limitation of those studies is that they focused only on representation of medical codes and did not utilize other sources of information from EHR data. Henriksson et al. applied word2vec to learn the vector representations of medical codes and words in clinical notes separately, and used both of them to predict adverse drug events [26, 27]. As they embed medical codes and words into two different spaces, their learned representations are not able to capture relationship between words and codes, which is exploited in our proposed method.

In this paper, we propose JointSkip-gram model: a novel joint learning scheme for word2vec model which embeds both diagnosis medical codes and words from clinical notes in the same continuous vector space.
The resulting representations capture not only similarity between codes or words themselves, but also similarity between codes and words. We believe many clinical tasks can be viewed as measuring similarity between codes and words. For example, text-based phenotyping $[12,13]$ is the process of discovering the most representative words for diagnostic medical concepts. On the other hand, given a collection of words, such as clinical notes, the automatic code assignment task [11] aims to automatically assign diagnosis and procedure medical codes and thus reduce human coding effort. In this paper we illustrate that it is possible to obtain representation of words and codes in the same vector space and that the resulting representations are very informative. To achieve this objective, directly applying word2vec and related algorithms may not be appropriate since codes and words are located in different parts of EHR and have different forms and properties. Our proposed model is designed to tackle the heterogeneous nature of EHR data and build a connection between medical codes and words in clinical notes.

In our experiments, we examined if our representations are able to discover meaningful text-based phenotypes for different medical concepts. We compared our proposed model with Labeled LDA [28], a supervised counterpart of Latent Dirichlet Allocation (LDA) [29], which has been applied previously to clinical data analysis [30-32]. The results show that our representations indeed capture the relationship between words and codes. In comparison to our previous study [21], we also show that our method is able to identify common medicines and treatments for different diseases. We also construct patient representations and test the predictive power of the representations on the task of predicting patient diagnosis of the next visit given information from the current visit. The results show that representations learned by our approach outperform several baseline methods.

\section{Methods}

After formulating the problem setup we overview Skip-gram [14], the architecture contained in word2vec toolkit designed for learning representations of natural language words, which is also the basis of our method. Then we explain the proposed JointSkip-gram model.

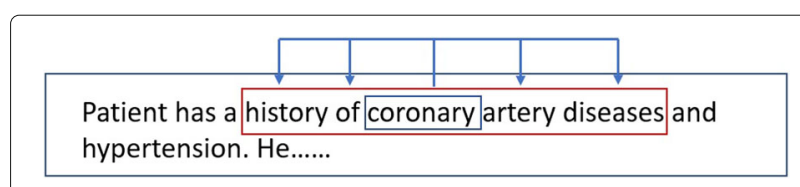

Fig. 1 The framework of Skip-gram. Each word is used to predict its neighbours in a small context window. In this example the size of context window is 2 


\section{Basic problem setup}

Let us assume we are given a collection of patient visits. Each visit $S$ is a pair $(D, N)$, where $D$ is an unordered set of medical diagnosis codes $\left\{c_{1}, c_{2}, c_{3} \ldots, c_{n}\right\}$ summarizing health condition of a patient and $N$ is an ordered sequence of words from clinical notes recorded during the visit $\left(w_{1}, w_{2}, w_{3} \ldots, w_{m}\right)$. We denote the size of the code vocabulary $C$ as $|C|$ and the size of the word vocabulary $W$ as $|W|$.

\section{Preliminary: Skip-gram}

Figure 1 summarizes the Skip-gram framework. Given a sequence of words $\left(w_{1}, w_{2}, w_{3} \ldots, w_{m}\right)$, Skip-gram sequentially scans it. For every scanned word $w_{i}$, called the target word, the log-likelihood of the words within its neighborhood (e.g., a window of a predefined size $q$ ) is calculated as

$$
\sum_{i-q \leq j \leq i+q, j \neq i} \log p\left(w_{j} \mid w_{i}\right)
$$

where $p\left(w_{j} \mid w_{i}\right)$ is the conditional probability of seeing word $w_{j}$ as context of target word $w_{i}$. It is defined as a softmax function

$$
p\left(w_{j} \mid w_{i}\right)=\frac{e^{V_{w_{i}} \cdot U_{w_{j}}}}{\sum_{w_{k} \in W} e^{V_{w_{i}} \cdot U_{w_{k}}}}
$$

where $V_{w_{i}}$ is a $T$-dimensional vector providing the input representation of target word $w_{i}$ and $U_{w_{j}}$ is a $T$-dimensional vector providing the context representation of context word $w_{j}$. Skip-gram results in two matrices: the input word matrix $V \in \mathbb{R}^{|W| \times T}$ and the context word matrix $U \in \mathbb{R}^{|W| \times T}$. The obtained input word representation $V_{w_{i}}$ is typically used as word representation in downstream predictive or descriptive tasks.

To learn vector representation of words from the vocabulary, a stochastic gradient algorithm is used to maximize the objective function (1).
Maximizing (1) is computationally expensive since the denominator $\sum_{w_{k} \in W} e^{V_{w_{i}} U_{w_{k}}}$ in (2) sums over all words $w_{k} \in W$. As a computationally efficient alternative of (1), Mikolov et al. proposed the skip-gram with negative sampling (SGNS) [14], which replaces $\log p\left(w_{j} \mid w_{i}\right)$ in (1) with the sum of two logarithmic probabilities as follows. For scanned word $w_{i}$, the objective function becomes

$$
\sum_{\substack{i-q \leq j \leq i+q \\ j \neq i}}\left(\log p\left(w_{i}, w_{j}\right)+\sum_{w_{N} \in W_{n e g}} \log \left(1-p\left(w_{i}, w_{N}\right)\right)\right)
$$

where probability $p\left(w_{i}, w_{j}\right)$ is defined as sigmoid function $\sigma\left(V_{w_{i}} \cdot U_{w_{j}}\right)$ :

$$
p\left(w_{i}, w_{j}\right)=\sigma\left(V_{w_{i}} \cdot U_{w_{j}}\right)=\frac{1}{1+e^{-V_{w_{i}} \cdot U_{w_{j}}}}
$$

and $W_{\text {neg }}=\left\{w^{k} \sim P_{w} \mid k=1, \ldots, K\right\}$ is the set of so-called "negative words" that are sampled from the marginal distribution $P_{w}$ of words. $K$ is a hyperparameter determining the number of negative words generated with each context word. The assumption is that words sampled from the marginal distribution are less likely to co-occur as context of the target word. The first term of (3) is the probability that two words occur as target and context in the data set, while the second term of (3) is the probability that a target word and "negative words" in $W_{\text {neg }}$ are not observed co-occurring in the dataset. By maximizing (3), the dot product between frequently co-occurring words would become large while the dot product between rarely co-occurring words would become small. In other words, in the resulting $T$-dimensional vector space, the related words will be placed in the vicinity of each other, such that their cosine similarity is high.

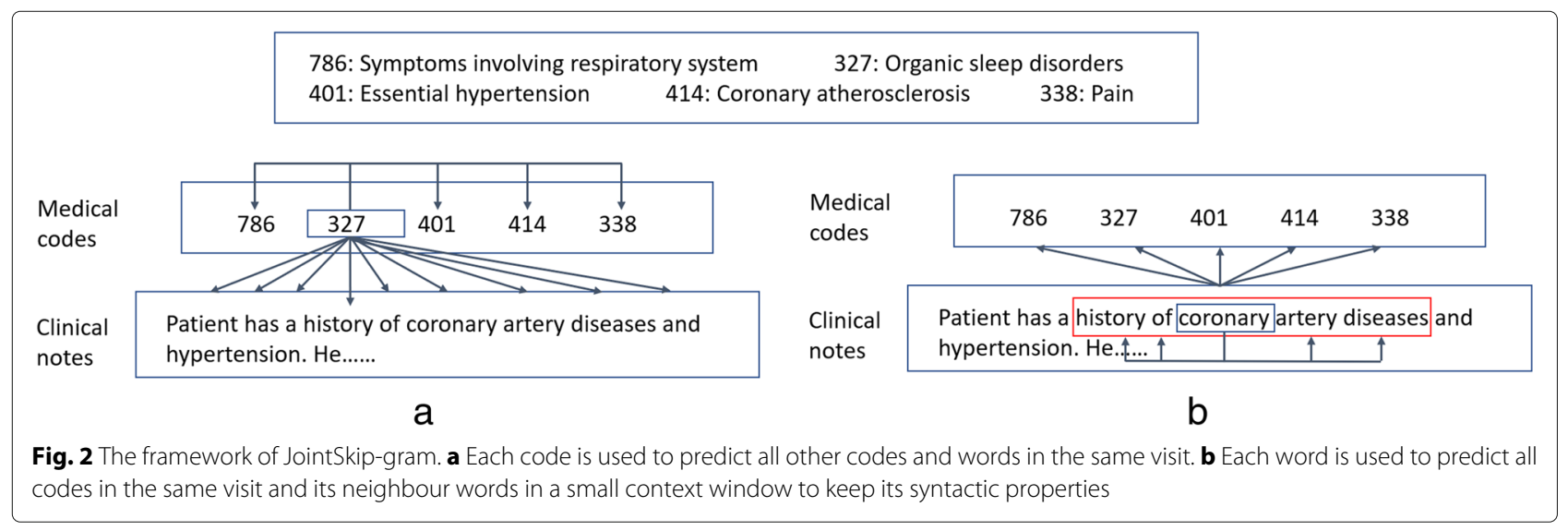




\section{Proposed model: JointSkip-gram}

In the Skip-gram model, each scanned word is used to predict probability of its neighboring words in the sequence. However, in the electronic health records each visit consists of clinical notes, which are ordered sequences of words, and medical codes, which are sets. We are interested in jointly learning vector representation of words and codes in the same vector space. Both medical codes and clinical notes describe condition and treatment of a patient and they are closely related. For example, if a patient is assigned ICD-9 code "174" (female breast neoplasm), the corresponding clinical notes are likely to mention surgery (e.g, mastectomy or lumpectomy). To derive JointSkip-gram, we first need to define context of each word and each code.

Since the codes are unordered, we define the context of target code $c_{i}$ as all other codes in the same visit, as well as all words in the clinical note. Thus, as shown in Fig. 2a, in JointSkip-gram, every scanned code $c_{i}$ is used to predict other codes in $D$ and all words in $N$. The log-likelihood of code $c_{i}$ can be expressed as

$$
\sum_{\substack{1 \leq j \leq n \\ j \neq i}} \log p\left(c_{j} \mid c_{i}\right)+\sum_{1 \leq j \leq m} \log p\left(w_{j} \mid c_{i}\right)
$$

Similarly to Skip-gram, the probabilities $p\left(c_{j} \mid c_{i}\right)$ and $p\left(w_{j} \mid c_{i}\right)$ are defined as softmax functions

$$
p\left(c_{j} \mid c_{i}\right)=\frac{e^{V_{c_{i}} \cdot U_{c_{j}}}}{\sum_{c_{k} \in C} e^{V_{c_{i}} \cdot U_{c_{k}}}}
$$

and

$$
p\left(w_{j} \mid c_{i}\right)=\frac{e^{V_{c_{i}} \cdot U_{w_{j}}}}{\sum_{w_{k} \in W} e^{V_{c_{i}} \cdot U_{w_{k}}}}
$$

For words in clinical notes we define two types of contexts. One consists of neighboring words in the note. Another consists of all codes in the medical code set. Thus, as shown in Fig. 2b, for scanned word $w_{i}$ in $N$ JointSkip-gram uses words within a window of a predefined size $q$ as its context words. It also uses all codes in $D$ as its context codes. The resulting log-likelihood of word $w_{i}$ can be expressed as

$$
\sum_{\substack{i-q \leq j \leq i+q \\ j \neq i}} \log p\left(w_{j} \mid w_{i}\right)+\sum_{1 \leq j \leq n} \log p\left(c_{j} \mid w_{i}\right)
$$

$$
p\left(w_{j} \mid w_{i}\right)=\frac{e^{V_{w_{i}} \cdot U_{w_{j}}}}{\sum_{w_{k} \in W} e^{V_{w_{i}} \cdot U_{w_{k}}}}
$$

and

$$
p\left(c_{j} \mid w_{i}\right)=\frac{e^{V_{w_{i}} \cdot U_{c_{j}}}}{\sum_{c_{k} \in C} e^{V_{w_{i}} \cdot U_{c_{k}}}}
$$

Maximizing the sum of objective functions (5) and (8) over the whole data set of visits is computationally expensive since in (6), (7), (9) and (10), the denominators sum over all words in $W$ and all codes in $C$. Similar to SGSN [14], we use a computationally cheaper algorithm that relies on negative sampling. Instead of calculating the softmax function, the negative sampling approach uses computationally inexpensive sigmoid function to represent the probability that a word or a code is within a context of a target word or a code. For each scanned code $c_{i}$, the negative sampling objective function becomes

$$
\begin{aligned}
& \sum_{\substack{1 \leq j \leq n \\
j \neq i}}\left(\log p\left(c_{i}, c_{j}\right)+\sum_{c_{N} \in C_{\text {neg }}} \log \left(1-p\left(c_{i}, c_{N}\right)\right)\right) \\
& +\sum_{1 \leq j \leq m}\left(\log p\left(c_{i}, w_{j}\right)+\sum_{w_{N} \in W_{\text {neg }}} \log \left(1-p\left(c_{i}, w_{N}\right)\right)\right)
\end{aligned}
$$

where

$$
p\left(c_{i}, c_{j}\right)=\sigma\left(V_{c_{i}} \cdot U_{c_{j}}\right)=\frac{1}{1+e^{-V_{c_{i}} \cdot U_{c_{j}}}}
$$

and

$$
p\left(c_{i}, w_{j}\right)=\sigma\left(V_{c_{i}} \cdot U_{w_{j}}\right)=\frac{1}{1+e^{-V_{c_{i}} \cdot U_{w_{j}}}}
$$

$C_{\text {neg }}=\left\{c^{k} \sim P_{c} \mid k=1, \ldots, K\right\}$ is the set of "negative codes" that are sampled from marginal distribution $P_{c}$ of codes and $W_{\text {neg }}=\left\{w^{k} \sim P_{w} \mid k=1, \ldots, K\right\}$ is the set of negative words that are sampled from a marginal distribution $P_{w}$ of words, where $K$ is the number of negative samples.

Similarly, for each scanned word $w_{i}$, the negative sampling objective criterion becomes:

$$
\begin{aligned}
& \sum_{\substack{i \leq j \leq i+q \\
j \neq i}}\left(\log p\left(w_{i}, w_{j}\right)+\sum_{w_{N} \in W_{\text {neg }}} \log \left(1-p\left(w_{i}, w_{N}\right)\right)\right) \\
& +\sum_{1 \leq j \leq n}\left(\log p\left(w_{i}, c_{j}\right)+\sum_{c_{N} \in C_{\text {neg }}} \log \left(1-p\left(w_{i}, c_{N}\right)\right)\right)
\end{aligned}
$$

in which 
where

$$
p\left(w_{i}, w_{j}\right)=\sigma\left(V_{w_{i}} \cdot U_{w_{j}}\right)=\frac{1}{1+e^{-V_{w_{i}} \cdot U_{w_{j}}}}
$$

and

$$
p\left(w_{i}, c_{j}\right)=\sigma\left(V_{w_{i}} \cdot U_{c_{j}}\right)=\frac{1}{1+e^{-V_{w_{i}} \cdot U_{c_{j}}}}
$$

$C_{\text {neg }}$ and $W_{\text {neg }}$ are the same as in (11). By maximizing (14), the probabilities $p\left(w_{i}, w_{j}\right)$ and $p\left(w_{i}, c_{j}\right)$ of related words and codes will be large.

Similarly to Skip-gram, stochastic gradient descent algorithm is applied in jointSkip-gram to learn vector representations of codes and words that maximize (11) and (14). The input vector representation matrix $V$ is used as the resulting representation of words and codes. Since we jointly learn vector representations of codes and words, matrices $V \in \mathbb{R}^{(|W|+|C|) \times T}$ and $U \in \mathbb{R}^{(|W|+|C|) \times T}$ include representations of both words and codes. In the resulting vector space, similarity of two vectors is measured using cosine similarity. The vectors of similar codes or words should be close to each other. Since JointSkip-gram represents codes and words in the same vector space, the words related to a given medical code should be placed in vicinity.

\section{Results}

\section{Dataset description}

MIMIC-III Dataset: The MIMIC-III Critical Care Database [33] is a publicly-available database which contains de-identified health records of 46,518 patients who stayed in the Beth Israel Deaconess Medical Center's Intensive Units from 2001 to 2012. Each visit in the dataset contains both structured health records data and free text clinical notes.

We used EHR data from all patients in the dataset. The total number of patient visits in MIMIC-III is 58,597 . On average, each patient had 1.26 visits, 38,991 patients had a single visit, 5151 had two visits, and 2376 patients had 3 or more visits. The average number of the recorded ICD-9 diagnosis codes per visit is 11 and the average number of words in clinical notes is 7898 . For each patient visit, we extracted all diagnosis codes and all clinical notes.

Preprocessing: For each EHR in the dataset we are only focusing on the clinical notes and ICD-9 diagnosis codes. Each clinical note was preprocessed in the following way. All digits and stop words were removed. The typos were filtered using a standard English vocabulary in PyEnchant, a Python library for spell checking. For representation learning, rare words were filtered out since they do not appear often enough to obtain good quality representations. Therefore, all words whose frequency is less than 50 were removed. The resulting number of unique words was 14,302. Furthermore, the total number of unique ICD-9 diagnosis codes in MIMIC-III is 6984. Codes whose frequency is less than 5 were removed. This reduced the number of codes to 3874 . Since some codes were still relatively rare for learning meaningful representations, we exploited the hierarchical tree structure of ICD-9 codes and grouped them by their first three digits. For example, ICD-9 codes "2901" (presenile dementia), "2902" (senile dementia with delusional or depressive) and "2903" (senile dementia with delirium) were grouped into a single code "290" (dementias). The size of the final code vocabulary was 752 .

Training and Test Patients: We randomly split the patients into training and test sets. All 38,991 patients with a single visit were placed in the training set. Of the 7527 patients with 2 or more visits, we randomly assigned $80 \%$ of them (6015 patients) to the training set and $20 \%$ of them (1512 patients) to the test set. The whole training set was used for learning of vector representations. We excluded patients with only a single visit for the task of next visit prediction because this task requires patients to have at least two visits.

\section{Training JointSkip-gram model}

EHRs of patients from the training set were used to learn our JointSkip-gram model. For each visit we created a $(D, N)$ pair. There were 54,965 such pairs in the training data. The size $T$ of vectors representing codes and words was set to 200. Stochastic gradient algorithm with negative sampling maximizing (11) and (14) was set to loop through all the training data 40 times because we empirically observed that it was sufficient for the algorithm to converge. The number of negative samples was set to 5 and the size of the window for word context in the clinical notes was set to 5 . As a result, each of the 7898 words and 752 ICD-9 codes were represented as 200-dimensional vectors in a joint vector space. Before applying JointSkipgram model, we used a small fraction $(\sim 10 \%)$ of clinical notes to pretrain vector representations of words only, as we observed that this improves our final representations.

To evaluate the quality of vector representations, we performed two types of experiments: (1) phenotype and treatment discovery by evaluating associations between codes and words in the vector space, (2) testing the predictive power of the vector representations on the task of predicting medical codes of the next visit.

\section{Phenotype discovery}

Text-based phenotype discovery can be viewed as finding words representative of medical codes. For a given ICD9 diagnosis code, we retrieved its nearest 15 words in the vector space. If successful, the neighboring words should be clinically relevant to the ICD-9 code. 
As an alternative to JointSkip-gram, we used labeled latent Dirichlet allocation (LLDA) [28], a supervised version of LDA [29]. In LLDA, there is a one-to-one correspondence between topics and labels. LLDA assumes there are multiple labels associated with each document and assigns each word a probability that it corresponds to each label. LLDA can be naturally adapted to our case by treating medical codes as labels and clinical notes as documents. For a given ICD-9 diagnosis code we retrieved 15 words with the highest probabilities and compared those words with the 15 words obtained by JointSkip-gram.

We consulted domain experts about quality of the extracted phenotypes. First, we selected 6 diverse ICD-9 codes from MIMIC-III that cover both acute and chronic diseases and both common and less common conditions. The 6 ICD-9 codes are listed in Table 1, together with their description and frequency in the training set. Table 1 shows the list of 15 closest words by both methods to the 6 ICD-9 codes. For each ICD-9 diagnosis code, we presented the two lists in a random order to a medical expert and asked two questions: (1) which list is a better representative of the diagnosis code, and (2) which words in each list are not highly related to the given diagnosis code. We recruited four physicians from the Fox Chase Cancer Center as medical experts for the evaluation.

The evaluation results are summarized in Table 2. As could be seen, all 4 experts agreed that JointSkip-gram words better represent ICD-9 codes 570, 348, and 311. For the remaining 3 codes $(174,295,042)$, the experts were split, but in no case the majority preferred the LLDA words. By considering the average number of words deemed unrelated by the experts, the experts found that JointSkip-gram was superior to LLDA for all 6 ICD-9 diagnosis codes.

For ICD-9 code "570" (acute liver failure), JointSkipgram finds "liver", "hepatic", "cirrhosis", which are directly related to acute liver failure. Remaining words in the JointSkip-gram list are mostly indirectly related to liver failure, such as "alcoholic", which explains one of the primary reasons for liver damage. On the other hand, LLDA captured a few related words, as evidenced by an average of 9.25 words that experts found unrelated. Among those unrelated words we find "cooling", "sun", "arctic", "rewarmed", "cooled", "rewarming", "coded", "continue", and "prognosis".

For ICD-9 code "174" (female breast cancer), "295" (Schizopherenic disorders) and "042" (HIV), both JointSkipgram and LLDA find highly related words. One of our experts commented that several words found by JointSkip-gram are diseases which are likely to cooccur with the given disease. For example, JointSkip-gram finds "melanoma" for female breast cancer and "herpes", "chlamydia", "syphilis" for HIV. This suggests that JoinSkip-gram captures the hidden relationships between
Table 1 Most important 15 words (ranked by importance) for ICD-9 codes "570", "174", "295", "348", "311", "042"

\begin{tabular}{|c|c|c|c|}
\hline \multicolumn{2}{|c|}{570 (Acute liver failure, 1067) } & \multicolumn{2}{|c|}{174 (Female breast cancer, 139) } \\
\hline JointSkip-gram & LLDA & JointSkip-gram & LLDA \\
\hline Liver & Arrest & Metastatic & Breast \\
\hline Hepatic & Pea & Mets & Pres \\
\hline Cirrhosis & Cooling & Cancer & Mastectomy \\
\hline Rising & Sun & Breast & Flap \\
\hline Markedly & Arctic & Metastases & Mets \\
\hline Shock & Rewarmed & Malignant & $\mathrm{Ca}$ \\
\hline Lactate & Cooled & Metastasis & Cancer \\
\hline Encephalopathy & Atrophine & Oncologist & Metastatic \\
\hline Amps & Dopamine & Oncology & Chemotherapy \\
\hline Picture & Rewarming & Chemotherapy & Malignant \\
\hline Rise & Cardiac & Infiltrating & Oncologist \\
\hline Elevated & Coded & Palliative & Polumoprhic \\
\hline Cirrhotic & Continue & Tumor & Reversible \\
\hline Bicarb & Prognosis & Melanoma & Mastectomies \\
\hline AQlcoholic & Ems & Mastectomy & Crisis \\
\hline $\begin{array}{l}295 \\
\text { (Schizophrenic }\end{array}$ & & $\begin{array}{l}348 \text { (Conditons } \\
\text { of brain, 3781) }\end{array}$ & \\
\hline
\end{tabular}

disorders, 691)

JointSkip-gram LLDA

JointSkip-gram LLDA

\begin{tabular}{llll} 
Schizophrenia & Schizophrenia & Hemorrhagic & Arrest \\
Psych & Paranoid & Herniation & Herniation \\
Bipolar & Psych & Temporal & Unresponsive \\
Suicide & Psychiatric & Cerebral & Corneal \\
Psychiatry & Disorders & Brain & Pupils \\
Kill & Personality & Hemorrhage & Brain \\
Paranoid & Hiss & Parietal & Cooling \\
Ideation & Guardian & Ganglia & Posturing \\
Psychiatrist & Psychiatry & Occipital & Head \\
Hallucinations & Hypothyroidism & Extension & Nemorrhage \\
Psychosis & Home & Surrounding & Noxious \\
Personality & Aloe & Head & Family \\
Sitter & Arrest & Effacement & Prognosis \\
Disorder & Pt & Ataxia & Pea \\
Abuse & Unresponsive & Burr & Gag \\
311 (Depressive & & $\mathbf{0 4 2}$ (HIV, 538) & \\
disorder, 3431) & & & JointSkip-gram \\
JointSkip-gram & LLDA & LLD \\
Patient & Depression & Aids & Aids \\
Abuse & Tablet & Viral & Immunodeficiency \\
Hallucinations & Blood & Fungal & Virus \\
Withdrawal & Daily & Opportunistic & Human \\
\hline & & & \\
\hline
\end{tabular}


Table 1 Most important 15 words (ranked by importance) for ICD-9 codes "570", "174", "295", "348", "311", "042" (Continued)

\begin{tabular}{llll}
\hline Ingestion & Campus & Bacterial & Viral \\
Questionable & Mg & Disseminated & Load \\
Thiamine & Garage & Immuno-deficiency & Cooling \\
Remote & Capsule & Tuberculosis & Partner \\
Alcohol & Building & Organisms & Acyclovir \\
Significant & Parking & Herpes & Thrush \\
Overdose & One & Undetectable & Fevers \\
Prior & Discharge & Acyclovir & Induced \\
Apparent & Normal & Detectable & Antigen \\
Depression & East & Chlamydia & Pneumonia \\
Although & Coherent & Syphilis & Blanket \\
\hline
\end{tabular}

Disease description and frequency are listed in the brackets

diseases, which could make it suitable for understanding of comorbidities.

For code "311" (depressive disorder), both JointSkipgram and LLDA had difficulties in finding related words. According to feedback from one of our experts, "abuse", "hallucinations", "alcohol", "overdose", "depression" and "thiamine" (note: depression is a common symptom of thiamine deficiency) found by JointSkip-gram are related to the disease, while only "depression", "tablet", "capsule" found by LLDA are recognizably related to depression. We hypothesize that for common diseases (e.g, "depression" and "hypertension"), which are rarely the primary diagnosis or a major factor in deciding an appropriate treatment of the main condition, physicians rarely discuss them in clinical notes. Thus, it is difficult for any algorithm to discover words from clinical notes related to such diagnoses.

\section{Treatment discovery}

In our preliminary study [21], we used PyEnchant standard English vocabulary to filter out the typos in clinical notes. However, there are many nonstandard English

Table 2 Evaluation results by clinical experts

\begin{tabular}{|c|c|c|c|c|c|c|}
\hline \multicolumn{7}{|c|}{ \# of experts who think the method is better than the other } \\
\hline ICD-9 codes & 570 & 174 & 295 & 348 & 311 & 042 \\
\hline JointSkip-gram & 4 & 2 & 3 & 4 & 4 & 2 \\
\hline LLDA & 0 & 2 & 1 & 0 & 0 & 2 \\
\hline \multicolumn{7}{|c|}{ Average \# of unrelated words across experts } \\
\hline ICD-9 codes & 570 & 174 & 295 & 348 & 311 & 042 \\
\hline JointSkip-gram & 2.25 & 0.75 & 0.75 & 1.25 & 3.25 & 0.75 \\
\hline LLDA & 9.25 & 1.75 & 3 & 3.75 & 6.5 & 2.75 \\
\hline
\end{tabular}

Table 3 Most important 15 words (including nonstandard English words) (ranked by importance) for ICD-9 codes "570"

\begin{tabular}{|c|c|}
\hline \multicolumn{2}{|c|}{ ICD-9: 570 (Acute liver failure) } \\
\hline Word & Description \\
\hline liver & $\begin{array}{l}\text { An organ that produces biochemicals necessary for } \\
\text { digestion }\end{array}$ \\
\hline Renal & Relating to the kidneys \\
\hline Hepatorenal & $\begin{array}{l}\text { A life-threatening medical condition that consists of } \\
\text { rapid deterioration in kidney }\end{array}$ \\
\hline Crrt & $\begin{array}{l}\text { CRRT is a dialysis modality used to treat critically ill, } \\
\text { hospitalized patients }\end{array}$ \\
\hline Vasopressin & A hormone synthesized \\
\hline Shock & Shock liver is a condition defined as an acute liver injury \\
\hline Failure & Liver failure can occur gradually \\
\hline Levophed & Injection \\
\hline Ascites & Ascites is the abnormal buildup of fluid in the abdomen \\
\hline Oliguric & A urine output \\
\hline Pigtail & Pigtail drainage is used for liver abscess \\
\hline Transplant & liver transplant is a surgical procedure \\
\hline Rifaximin & Antibiotic \\
\hline Cirrhosis & Cirrhosis is a late stage of scarring (fibrosis) of the liver \\
\hline Hepatic & Relating to the liver. \\
\hline
\end{tabular}

Table 4 Most important 15 words (including nonstandard English words) (ranked by importance) for ICD-9 codes 174

\begin{tabular}{|c|c|}
\hline \multicolumn{2}{|c|}{ ICD-9: 174 (Female breast cancer) } \\
\hline Word & Description \\
\hline Xeloda & $\begin{array}{l}\text { A prescription medicine used to treat people with } \\
\text { cancer }\end{array}$ \\
\hline Tamoxifen & A medication that is used to prevent breast cancer \\
\hline Metastatic & $\begin{array}{l}\text { A pathogenic agent's spread from an primary site to a } \\
\text { different site }\end{array}$ \\
\hline Chemotherapy & A treatment by the use of chemical substances \\
\hline Cancer & $\begin{array}{l}\text { A disease in which abnormal cells divide } \\
\text { uncontrollably and destroy body tissue }\end{array}$ \\
\hline Carboplatin & It is used to treat ovarian cancer \\
\hline Onc & Abbreviations of oncologist \\
\hline Oncologist & A doctor who treats cancer \\
\hline Taxol & $\begin{array}{l}\text { It belongs to a class of chemotherapy drugs is the } \\
\text { abnormal buildup of fluid in the abdomen }\end{array}$ \\
\hline Chemo & Short form of chemotherapy \\
\hline Gemcitabine & Gemcitabine is an anti-cancer \\
\hline Mets & Abbreviations of metastasis \\
\hline Compazine & This medication is used to treat severe nausea \\
\hline Palliative & A medical care for relieving pain \\
\hline Metastases & The development of secondary malignant growths \\
\hline
\end{tabular}


terms used in medical notes to describe medical treatments, medicines, and diagnoses. These nonstandard words are not part of PyEnchant standard English vocabulary we used for preprocessing, but they could have important meaning. Hence, we repeated our experiments by including all words occurring more than 50 times. The resulting vocabulary increased to 33,336 unique words.

After running our Joint-Skipgram model on the new dataset, we looked at the representative words for each diagnosis code. Tables 3 and 4 show the 15 nearest clinical note words in the vector space to ICD9 codes " 570 " and " 174 ", respectively. We can observe that many retrieved words are different from those in Table 1 for codes " 570 " and "174". The words that also appear in Table 1 are marked with italic font in Tables 3 and 4.

A close look into Tables 3 and 4 reveals that most neighbors are specific medical terminology words describing drugs or treatments related to the diagnosis. For example, words "crrt", "levophed", "rifaximin", and "transplant" in Table 3, are related to treatment of acute liver failure. Similarly, words "xcloda", "tamoxifen", "carboplatin", "taxol", "compazine" in Table 4 are related to cancer treatment. Therefore, including nonstandard words in our vocabulary enabled us to connect specialized medical terms with particular ICD-9 diagnosis codes.

\section{Predictive evaluation}

In another group of experiments we constructed patient representations and evaluated quality of the vector representations of words and medical codes through predictive modeling. We adopted the evaluation approach used in [34], which predicts medical codes of the next visit given the information from the current visit. Specifically, given two consecutive visits of a patient, we used information of the first visit (i.e., medical codes and clinical notes) to predict medical codes assigned during the second visit. In the previous work on this topic, the authors of [23, 34, 35] used medical codes as features for prediction. In our evaluation, we used both medical codes and clinical notes to create predictive features. To generate a feature vector for the first visit, we found the average JointSkip-gram vector representation of the diagnosis codes and the average JointSkip-gram vector representation of the words used in clinical notes. Then, we concatenated those two averaged vectors. We call this method Concatenation-JointSG and compare it with the following five baselines:

Concatenation-One: The one-hot vector of medical codes and the one-hot vector of clinical notes for a given visit were concatenated. In the one-hot vector of each visit, words and codes which occur in the visit were encoded as 1 , otherwise they were encoded as 0 .
SVD: Singular vector decomposition (SVD) was applied to Concatenation-One representations to generate dense representations of visits.

LDA: Using latent Dirichlet allocation (LDA) [29], each document was represented as a topic probability vector. This vector was used as the visit representation. To apply LDA, for each visit we created a document that consists of concatenation of a list of medical diagnosis codes and clinical notes. We note that LLDA is not suitable for this task since its topics only contain words.

Codes-JointSG: To evaluate the predictive power of medical codes, we created features for a visit as the average JointSkip-gram vector representation of the diagnosis codes.

Words-JoinSG: To evaluate the predictive power of clinical notes, we created features for a visit as the average JointSkip-gram vector representation of the words in clinical notes.

To compare vector representations obtained by JointSkip-gram and Skip-gram, we also trained Skip-gram on clinical notes and on medical codes separately. The resulting vector representations are not in the same vector space. We used Skip-gram representations to construct 3 more groups of features:

Codes-SG: The features for a visit were the average Skip-gram vector representation of the diagnosis codes.

Words-SG: The features for a visit were the average Skip-gram vector representation of the words in clinical notes.

Concatenation-SG: We concatenated the features from Codes-SG and Words-SG.

Given a set of features describing the first visit, we used softmax to predict medical codes of the second visit. Let us assume the feature vector of the first visit is $x_{t}$, the size of code vocabulary is $|C|$ and $Z \in \mathbb{R}^{\left(|C| \times\left|x_{t}\right|\right)}$ is the weight matrix of softmax function. The probability that the next visit $y_{t+1}$ contains medical code $c_{i}$ is calculated as

$$
p\left(y_{t+1}\left(c_{i}\right)=1\right)=\frac{e^{Z_{i} \cdot x_{t}}}{\sum_{c_{k} \in C} e^{Z_{k} \cdot x_{t}}}
$$

Table 5 Performance of predicting medical codes of the next visit

\begin{tabular}{llll}
\hline Model & Top-20 recall & Top-30 recall & Top-40 recall \\
\hline Concatenation-One & $0.489 \pm 0.004$ & $0.590 \pm 0.004$ & $0.661 \pm 0.004$ \\
SVD & $0.478 \pm 0.004$ & $0.588 \pm 0.004$ & $0.652 \pm 0.004$ \\
LDA & $0.431 \pm 0.004$ & $0.530 \pm 0.004$ & $0.605 \pm 0.004$ \\
Codes-JointSG & $0.499 \pm 0.003$ & $0.592 \pm 0.003$ & $0.662 \pm 0.003$ \\
Words-JointSG & $0.437 \pm 0.004$ & $0.536 \pm 0.004$ & $0.609 \pm 0.004$ \\
Concatenation-JointSG & $\mathbf{0 . 5 0 6} \pm \mathbf{0 . 0 0 3}$ & $\mathbf{0 . 5 9 9} \pm \mathbf{0 . 0 0 3}$ & $\mathbf{0 . 6 7 0} \pm \mathbf{0 . 0 0 3}$
\end{tabular}

The average and standard error of Top- $k$ recall $(k=20,30,40)$ are provided 
We use Top-k recall [34] to measure the predictive performance, because it mimics the behavior of doctors who list the most probable diagnoses upon observation of a patient. For each visit, softmax recommends $k$ codes with the highest probabilities and Top- $\mathrm{k}$ recall is calculated as

Top-k recall $=\frac{\text { the number of true positives in } k \text { codes }}{\text { the number of all positives }}$

In the experiment, we tested Top-k recall when $k=20$, $k=30$, and $k=40$.

Training details: To create features for all proposed models (Skip-gram, JointSkip-gram, LDA, SVD), we used the training set. To train the Skip-gram model, we used 40 iterations, 5 negative samples, and the window size 5 (the same as for JointSkip-gram). For SVD and LDA, we set the maximum number of iterations to 1000 to guarantee convergence. For JointSkip-gram, Skip-gram, SVD and LDA, we set the dimensionality of feature vectors to 200 .

To train the softmax model, we created the labeled set using only patients with 2 or more visits. We sort all visits of each such patient by the admission time. Given two consecutive visits, we use the former to create features and the latter to create the labels. As a result, the labeled set used to train the softmax model had 9955 labeled examples and the test set had 2489 labeled examples. The softmax model for prediction was trained for 100 epochs using a stochastic gradient algorithm to minimize the categorical cross entropy loss.

Table 5 shows the performance of softmax models that use different sets of features. A model using Concatenation-JointSG features outperformed other baselines on all three Top-k measures.
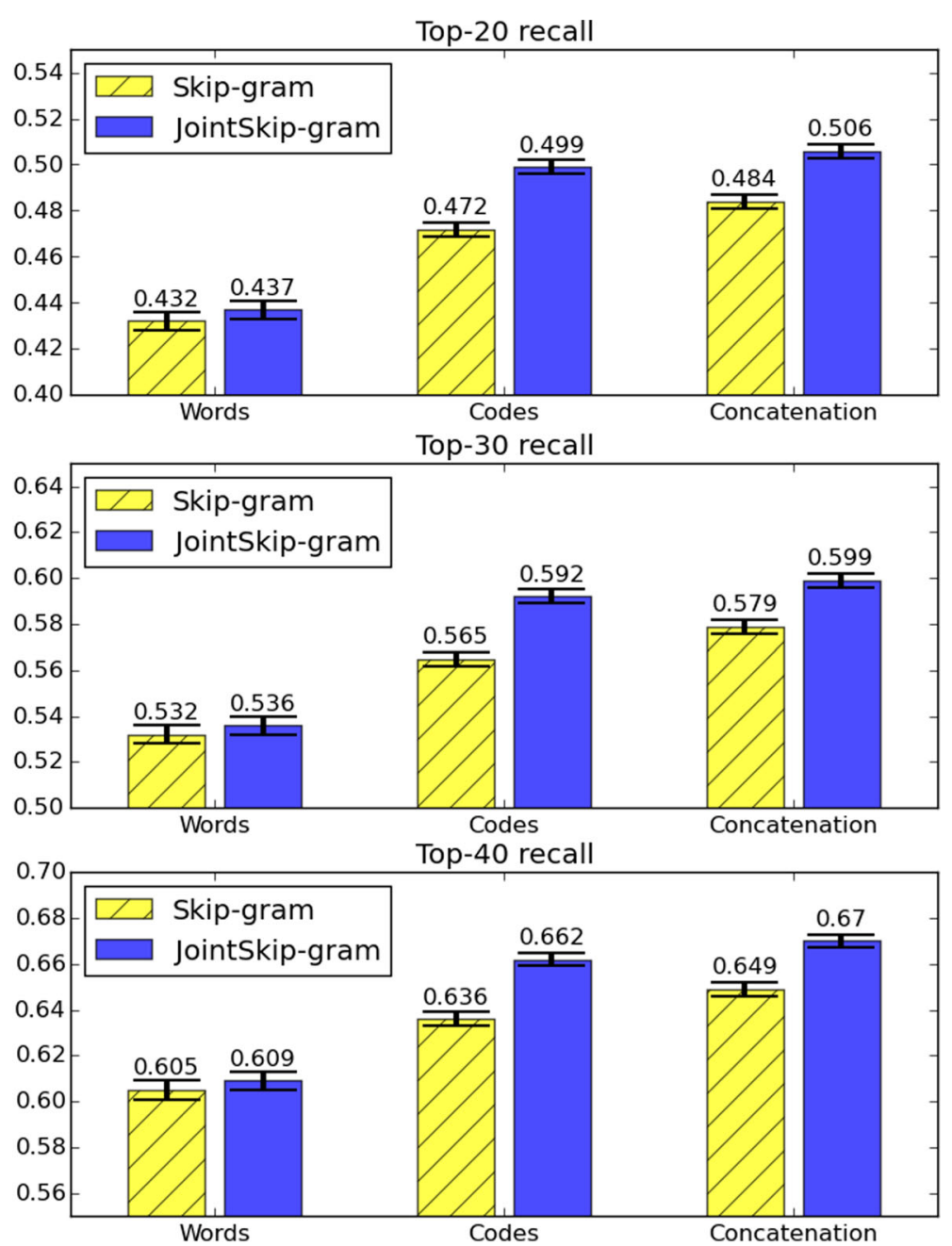

Fig. 3 Top-k recall ( $k=20,30$ and 40) for JointSkip-gram and Skip-gram. The error bars indicate the standard error 


\section{Discussion}

\section{Predictive evaluation analysis}

The results in Table 5 not only show the advantage of our model, but also demonstrate that both medical codes and clinical notes in Concatenation-JointSG contributed to the prediction of future visit, since using the concatenation of word representations and code representations outperformed both Codes-JointSG and Words-JointSG. While Codes-JointSG achieved considerably high recall, Words-JointSG performed relatively worse. The lower accuracy of Words-JointSG likely indicates that using the average of word vectors might not be the best strategy to use clinical note information. A future direction could be to use a neural network (NN) such as convolutional $\mathrm{NN}$ or recurrent $\mathrm{NN}$ to better capture information contained in clinical notes.

Figure 3 shows comparison between JointSkip-gram and Skip-gram features. From the figure, we can observe that features generated by JointSkip-gram outperformed those generated by Skip-gram. While the difference between Words-JointSG and Words-SG were not large, Codes-JointSG and Concatenation-JointSG significantly outperformed Codes-SG and Concatenation-SG, respectively. This strongly indicates that JointSkip-gram not only captures the relationship between medical codes and words, but also learns improved word and code representations.

\section{Limitations and future works}

One limitation of our work is that in processing step we removed words whose frequency are less than 50 and codes whose frequency are less than 5 . We also grouped all codes by their first three digits because rare codes are not statistically significant enough to learn meaningful representations. One way to use rare tokens is to exploit the domain knowledge such as subword information or hierarchical tree structure of medical codes.

The future work should consider applying joint representations to a broader range of tasks, such as cohort identification and automatic code assignment. It would also be interesting to explore more advanced prediction models such as deep neural networks.

\section{Conclusions}

In this paper, we proposed JointSkip-gram algorithm to jointly learn representation of words from clinical notes and diagnosis codes in EHR. JointSkip-gram exploits the relationship between diagnosis codes and clinical notes in the same visit and represents them in the same vector space. The experimental results demonstrate that the resulting code and word representation can be used to discover meaningful disease phenotypes. They also indicate that the representations learned by the joint model are useful for construction of patient features.

\section{Abbreviations \\ EHR: Electronic health record; ICD: International classification of diseases; LDA: Latent Dirichlet allocation; LLDA: Labeled latent Dirichlet allocation; NLP: natural language processing; NN: neural network; SVD: Singular vector decomposition}

\section{Acknowledgements}

Authors would like to thank the National Institute of Health for funding our research.

\section{Funding}

This work was supported by the National Institutes of Health grants R21CA202130 and P30CA006927. Publication costs were also funded by the National Institutes of Health grants R21CA202130 and P30CA006927.

\section{Availability of data and materials}

The data (MIMIC-III Dataset) used in our experiment can be obtained in https://mimic.physionet.org/. Researchers seeking to use the database must formally request access following the steps on their website.

\section{About this supplement}

This article has been published as part of BMC Medical Informatics and Decision Making Volume 18 Supplement 4, 2018: Selected articles from the IEEE BIBM International Conference on Bioinformatics \& Biomedicine (BIBM) 2017: medical informatics and decision making. The full contents of the supplement are available online at https://bmcmedinformdecismak.biomedcentral.com/ articles/supplements/volume-18-supplement-4.

\section{Authors' contributions}

TB and SV conceived the study and developed the algorithm. TB wrote the first draft of the manuscript. AKC conducted experiments for discovering treatment procedures. All authors participated in the preparation of the manuscript and approved the final version.

Ethics approval and consent to participate

Not applicable.

\section{Consent for publication}

Not applicable.

\section{Competing interests}

The authors declare that they have no competing interests.

\section{Publisher's Note}

Springer Nature remains neutral with regard to jurisdictional claims in published maps and institutional affiliations.

\section{Author details}

${ }^{1}$ Department of Computer \& Information Sciences, Temple University, Philadelphia, PA, USA. ${ }^{2}$ Fox Chase Cancer Center, Temple University, Philadelphia, PA, USA.

\section{Published: 12 December 2018}

\section{References}

1. Yan Y, Birman-Deych E, Radford MJ, Nilasena DS, Gage BF. Comorbidity indices to predict mortality from medicare data: results from the national registry of atrial fibrillation. Med Care. 2005;43:1073-7.

2. Krumholz HM, Wang Y, Mattera JA, Wang Y, Han LF, Ingber MJ, Roman S, Normand S-LT. An administrative claims model suitable for profiling hospital performance based on 30-day mortality rates among patients with heart failure. Circulation. 2006;113(13):1693-701.

3. Klabunde CN, Potosky AL, Legler JM, Warren JL. Development of a comorbidity index using physician claims data. J Clin Epidemiol. 2000;53(12):1258-67.

4. Levitan N, Dowlati A, Remick S, Tahsildar H, Sivinski L, Beyth R, Rimm A. Rates of initial and recurrent thromboembolic disease among patients 
with malignancy versus those without malignancy. Risk Anal Medicare Claims Data. Med (Baltimore). 1999;78(5):285-91.

5. Taylor Jr DH, Østbye T, Langa KM, Weir D, Plassman BL. The accuracy of medicare claims as an epidemiological tool: the case of dementia revisited. J Alzheimers Dis. 2009;17(4):807-15.

6. Schneeweiss S, Seeger JD, Maclure M, Wang PS, Avorn J, Glynn RJ. Performance of comorbidity scores to control for confounding in epidemiologic studies using claims data. Am J Epidemiol. 2001;154(9): 854-64.

7. Nattinger AB, Laud PW, Bajorunaite R, Sparapani RA, Freeman JL. An algorithm for the use of medicare claims data to identify women with incident breast cancer. Health Serv Res. 2004;39(6p1):1733-50.

8. Winkelmayer WC, Schneeweiss S, Mogun H, Patrick AR, Avorn J, Solomon DH. Identification of individuals with ckd from medicare claims data: a validation study. Am J Kidney Dis. 2005;46(2):225-32.

9. Warren JL, Klabunde CN, Schrag D, Bach PB, Riley GF. Overview of the seer-medicare data: content, research applications, and generalizability to the united states elderly population. Med Care. 2002;40:3-18.

10. Halpern Y, Horng S, Choi Y, Sontag D. Electronic medical record phenotyping using the anchor and learn framework. J Am Med Inform Assoc. 2016;23(4):731-40.

11. Wang Y, Patrick J. Mapping clinical notes to medical terminology at point of care. In: Proceedings of the Workshop on Current Trends in Biomedical Natural Language Processing. Stroudsburg: Association for Computational Linguistics; 2008. p. 102-3.

12. Pivovarov R, Perotte AJ, Grave E, Angiolillo J, Wiggins CH, Elhadad N. Learning probabilistic phenotypes from heterogeneous ehr data. J Biomed Inform. 2015;58:156-65.

13. Joshi S, Gunasekar S, Sontag D, Ghosh J. Identifiable phenotyping using constrained non-negative matrix factorization; 2016, pp. 17-41. arXiv preprint arXiv:1608.00704.

14. Mikolov T, Sutskever I, Chen K, Corrado GS, Dean J. Distributed representations of words and phrases and their compositionality. In: Advances in Neural Information Processing Systems. 2013. p. 3111-9.

15. Moen H, Ginter F, Marsi E, Peltonen L-M, Salakoski T, Salanterä S. Care episode retrieval: distributional semantic models for information retrieval in the clinical domain. In: BMC Medical Informatics and Decision Making, vol. 15. BioMed Central; 2015. p. 2. https://doi.org/10.1186/1472-694715-S2-S2.

16. Wu Y, Xu J, Jiang $M$, Zhang $Y, X u H$. A study of neural word embeddings for named entity recognition in clinical text. In: AMIA Annual Symposium Proceedings, vol. 2015. American Medical Informatics Association; 2015. p. 1326.

17. De Vine L, Zuccon G, Koopman B, Sitbon L, Bruza P. Medical semantic similarity with a neural language model. In: Proceedings of the $23 \mathrm{rd} \mathrm{ACM}$ International Conference on Conference on Information and Knowledge Management. New York: ACM; 2014. p. 1819-22.

18. Amunategui M, Markwell T, Rozenfeld Y. Prediction using note text: Synthetic feature creation with word2vec; 2015. arXiv preprint arXiv:1503.05123.

19. Ghassemi MM, Mark RG, Nemati S. A visualization of evolving clinical sentiment using vector representations of clinical notes. In: Computing in Cardiology Conference (CinC), 2015. IEEE; 2015. p. 629-32. http://doi.org/ 10.1109/CIC.2015.7410989.

20. Henriksson A. Representing clinical notes for adverse drug event detection. In: Proceedings of the Sixth International Workshop on Health Text Mining and Information Analysis. 2015. p. 152-8.

21. Bai T, Chanda AK, Egleston BL, Vucetic S. Joint learning of representations of medical concepts and words from ehr data. In: Bioinformatics and Biomedicine (BIBM), 2017 IEEE International Conference On. IEEE; 2017. p. 764-9. http://doi.org/10.1109/BIBM.2017. 8217752.

22. Choi Y, Chiu CY-I, Sontag D. Learning low-dimensional representations of medical concepts. AMIA Summits TransI Sci Proc. 2016;2016:41.

23. Choi E, Bahadori MT, Searles E, Coffey C, Thompson M, Bost J, TejedorSojo J, Sun J. Multi-layer representation learning for medical concepts. In: Proceedings of the 22nd ACM SIGKDD International Conference on Knowledge Discovery and Data Mining. New York: ACM; 2016. p. 1495-504.
24. Choi E, Schuetz A, Stewart WF, Sun J. Using recurrent neural network models for early detection of heart failure onset. J Am Med Inform Assoc. 2016;24(2):361-70.

25. Stojanovic J, Gligorijevic D, Radosavljevic V, Djuric N, Grbovic M, Obradovic Z. Modeling healthcare quality via compact representations of electronic health records. IEEE/ACM Trans Comput Biol Bioinforma (TCBB). 2017;14(3):545-54.

26. Henriksson A, Zhao J, Boström $H$, Dalianis H. Modeling electronic health records in ensembles of semantic spaces for adverse drug event detection. In: Bioinformatics and Biomedicine (BIBM), 2015 IEEE International Conference On. IEEE; 2015. p. 343-50. https://doi.org/10. 1109/BIBM.2015.7359705.

27. Henriksson A, Zhao J, Dalianis H, Boström H. Ensembles of randomized trees using diverse distributed representations of clinical events. BMC Med Inform Decis Mak. 2016;16(2):69.

28. Ramage D, Hall D, Nallapati R, Manning CD. Labeled Ida: A supervised topic model for credit attribution in multi-labeled corpora. In: Proceedings of the 2009 Conference on Empirical Methods in Natural Language Processing: Volume 1-Volume 1. Stroudsburg: Association for Computational Linguistics; 2009. p. 248-56.

29. Blei DM, Ng AY, Jordan MI. Latent dirichlet allocation. J Mach Learn Res. 2003;3(Jan):993-1022.

30. Chan KR, Lou X, Karaletsos T, Crosbie C, Gardos S, Artz D, Ratsch G. An empirical analysis of topic modeling for mining cancer clinical notes. In: Data Mining Workshops (ICDMW), 2013 IEEE 13th International Conference On. IEEE; 2013. p. 56-63. https://doi.org/10.1109/ICDMW. 2013.91.

31. Arnold CW, El-Saden SM, Bui AA, Taira R. Clinical case-based retrieval using latent topic analysis. In: AMIA Annual Symposium Proceedings, vol. 2010. American Medical Informatics Association; 2010. p. 26.

32. Ghassemi M, Naumann T, Doshi-Velez F, Brimmer N, Joshi R, Rumshisky A, Szolovits P. Unfolding physiological state: Mortality modelling in intensive care units. In: Proceedings of the 20th ACM SIGKDD International Conference on Knowledge Discovery and Data Mining. New York: ACM; 2014. p. $75-84$

33. Johnson AE, Pollard TJ, Shen L, Li-wei HL, Feng M, Ghassemi M, Moody B, Szolovits P, Celi LA, Mark RG. Mimic-iii, a freely accessible critical care database. Sci Data. 2016;3:160035.

34. Choi E, Bahadori MT, Schuetz A, Stewart WF, Sun J. Doctor ai: Predicting clinical events via recurrent neural networks. In: Machine Learning for Healthcare Conference; 2016. p. 301-18.

35. Esteban C, Staeck O, Baier S, Yang Y, Tresp V. Predicting clinical events by combining static and dynamic information using recurrent neural networks. In: Healthcare Informatics (ICHI), 2016 IEEE International Conference On. IEEE; 2016. p. 93-101.

\section{Ready to submit your research? Choose BMC and benefit from:}

- fast, convenient online submission

- thorough peer review by experienced researchers in your field

- rapid publication on acceptance

- support for research data, including large and complex data types

- gold Open Access which fosters wider collaboration and increased citations

- maximum visibility for your research: over $100 \mathrm{M}$ website views per year

At BMC, research is always in progress.

Learn more biomedcentral.com/submissions 